Author has nothing to disclose with regard to commercial support.

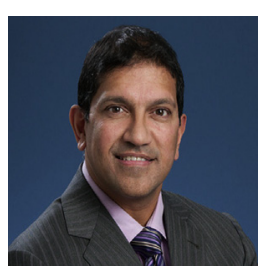

\section{HIGH-RISK SURGERY AS AN ALTERNATIVE TO HEART TRANSPLANT OR VENTRICULAR ASSIST DEVICE}

Reply to the Editor:

My coauthors and I appreciate the interest in our study and the comments of Drs Adhyapak and Parachuri. We agree that our study focused on a previously underinvestigated patient cohort, namely those who in addition to having severe left ventricular dysfunction met criteria for advanced surgical therapies such as transplant or ventricular assist device implantation. In contrast to many studies that simply used a left ventricular ejection fraction value less than $20 \%$ as an inclusion criterion, our study excluded those patients who had transient "hibernating" myocardium and those with stable cardiomyopathies not requiring advanced therapies. By focusing on those patients at high risk who met criteria for advanced therapies (by virtue of exercise testing), our results naturally show a higher mortality and morbidity than previous reports in a less defined population. Despite this higher risk, we were favorably impressed by the long-term outcomes of these patients. We agree with our colleagues that for "selected surgical candidates," conventional therapies, including revascularization, ventricular reconstruction, and valve repair, can have acceptable outcomes.

Vivek Rao, $M D, P h D$

Division of Cardiovascular Surgery

Peter Munk Cardiac Centre Toronto General Hospital Ottawa, Ontario, Canada

http://dx.doi.org/10.1016/j.jtcvs.2017.09.020

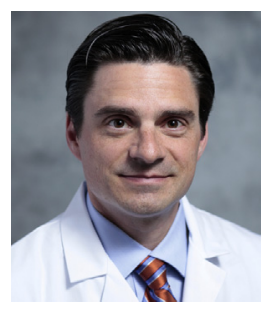

HIGH-RISK CARDIAC SURGERY, TRANSPLANT, $\left(\mathbb{Q}_{\text {cross }}\right.$ OR MECHANICAL SUPPORT? THE ANSWER CAN BE "ALL OF THE ABOVE"

\section{Reply to the Editor:}

Adhyapak and Parachuri have rightly focused on the most remarkable conclusion of the recent article by Kawajiri and colleagues ${ }^{1}$ - that advanced heart failure is not a contraindication to conventional cardiac surgery. This conclusion gives credence to the natural instincts of many cardiologists and surgeons to address existing pathology before considering replacement or assist strategies. Nevertheless, routinely incorporating an expected $12 \%(\mathrm{n}=16)$ in-hospital mortality into one's practice carries substantial reputational risks that may offset the benefits of taking on these types of cases-particularly in the era of public reporting. In the case of these 16 patients, 10 of whom died of low-output syndrome (LOS), we must look for innovative approaches to bring the survival curves more in line with the benchmarks for conventional surgery.

Recognizing that conventional surgery will fail approximately $7.5 \%$ of the time from LOS (on the basis of the data of Kawajiri and colleagues ${ }^{1}$ ), the challenge becomes predicting which patients are at risk for this complication and then finding new ways to support the failing ventricle. It may be that for these high-risk patients an either-or approach to surgical decision making is not the best strategy. Although concomitant valve procedures are often performed when left ventricular assist devices are inserted as bridge to transplant or as destination therapy, ${ }^{2}$ coronary artery disease is often left alone on the basis that myocardial recovery is unlikely. Nevertheless, implanting a left ventricular device in combination with revascularization or correcting structural diseases is an intriguing consideration for patients who may require months of reverse remodeling to regain adequate cardiac function. The addition of stem cell therapies and dischargeable short-term support devices to this type of approach could potentially further expand the pool of patients who are eligible for a "bridge to recovery" approach. This type of strategy obviously depends on improving the accuracy of preoperative calculation of the risk for LOS. Viability studies have often failed to consistently discriminate patients who will have myocardial recovery, ${ }^{3}$ and it seems likely that a more nuanced review of multiple imaging and patient characteristics is needed best to avoid patients who are not likely to recover after conventional surgery. In addition, the adverse event profiles and technical complexities of explanting the currently available devices are great enough to discourage many surgeons from considering this option as a routine strategy for avoiding LOS.

In addition to the prospective multicenter, randomized studies advocated by Adhyapak and Parachuri, our research should focus on both developing hardware that is more easily explantable and improving the accuracy of predicting myocardial recovery. As with most things in life and in cardiac surgery, the multiple-choice question of how to treat patients with end-stage heart failure may indeed have more than one correct answer.

David L. Joyce, $M D$

Director of Heart and Lung Transplant Froedtert and the Medical College of Wisconsin Milwaukee, Wis 